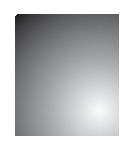

\title{
Relação entre Environmental Management AcCounting e InOVAÇÃO: APLICAÇÃo do MOdELO teórico de Ferreira, Moulang e Hendro (2010) em eMpresas do Rio Grande do Sul

\author{
Relationship between Environmental Management Accounting \\ and Innovation: application Ferreira, Moulang e Hendro (2010) \\ theoretical model in companies of Rio Grande do Sul
}

\begin{abstract}
Ilse Maria Beuren
Doutora em Controladoria e Contabilidade. Professora Titular do Departamento de Ciências Contábeis da Universidade Federal do

Paraná. Curitiba, PR. Brasil. E-mail: ilse.beuren@gmail.com

Vinícius Costa da Silva Zonatto

Doutor em Ciências Contábeis e Administração. Professor do Programa de Pós-Graduação em Ciências Contábeis da Universidade Regional de Blumenau. Blumenau, SC.Brasil.E-mail: viniciuszonatto@gmail.com
\end{abstract}

\section{Resumo}

O estudo objetiva identificar a relação entre práticas de contabilidade de gestão ambiental e inovação, fundamentado no modelo teórico de Ferreira, Moulang e Hendro (2010), em empresas industriais de grande porte estabelecidas no Rio Grande do Sul, Brasil. Pesquisa descritiva foi realizada por meio de levantamento em 21 empresas industriais selecionadas por acessibilidade, com aplicação de modelagem de equações estruturais Partial Least Squares (PLS). Os resultados mostram que nem todas as empresas pesquisadas adotam práticas de sistemas de Environmental Management Accounting. Também evidenciam associação positiva entre a estratégia prospectora e o tamanho da empresa, em relação ao uso de sistemas EMA. A estratégia prospectora influencia o esforço em pesquisa e desenvolvimento e em inovação de produtos e processos. As variáveis de inovação de processos e inovação de produtos apresentaram forte correlação. As evidências encontradas permitem concluir que há associação positiva entre uso de sistemas EMA e inovação nas empresas pesquisadas.

Palavras-chave: Contabilidade de Gestão Ambiental. Inovação. Empresas Industriais.

\section{Abstract}

The study aims to identify the relationship between the environmental management accounting practices and innovation, based on Ferreira, Moulang e Hendro (2010) theoretical model, in large industrial companies established in the State of Rio Grande do Sul, Brazil. Descriptive study was conducted by surveying in 21 industrial companies selected by accessibility, with application Partial Least Squares (PLS) structural equation modeling. The results show that not all companies surveyed adopt Environmental Management Accounting (EMA) practices. Also, indicate positive association between a prospector strategy and company size in relation to the use of EMA systems. The prospector strategy influences the effort in research and development and the innovation of products and processes. The variables of process innovation and product innovation were strongly correlated. The evidences allow to conclude that there is a positive association between use of EMA systems and innovation in the companies surveyed.

Keywords: Environmental Management Accounting. Innovation. Industrial Companies. 


\section{INTRODUÇÃo}

A preocupação com questões ambientais é crescente nas organizações e na literatura atual. Diversos são os autores que analisaram os efeitos das questões ambientais sobre as organizações em diferentes perspectivas, como investimentos em proteção ambiental (ROGERS; KRISTOF, 2003), passivos ambientais (MARTIN, 2007), evidenciação ambiental (MARSHALL; BROWN, 2003), desempenho ambiental e organizacional (BRAMER; BROOKS; PAVELIN, 2006), e a relação da contabilidade com questões de sustentabilidade. (BURRITT, 2004)

Apesar da crescente preocupação com esses temas, a contabilidade de gestão ambiental (Environmental Management Accounting - EMA) tem recebido pouca atenção por parte dos pesquisadores da área contábil (FERREIRA; MOULANG; HENDRO, 2010). De acordo com Burritt (2004), o propósito central da contabilidade de gestão ambiental é fornecer aos gestores informações relativas às atividades empresariais que afetam o meio ambiente, bem como analisar os impactos do ambiente sobre as atividades da corporação.

A pesquisa ambiental em contabilidade tem se desenvolvido rapidamente nos últimos anos (BURRITT, 2004; GIBSON; MARTIN, 2004; BURRITT; SAKA, 2006; MASANET-LLODRA, 2006; STANISKIS; STASISKIENE, 2006; FERREIRA; MOULANG; HENDRO, 2010). No entanto, a investigação sobre aspectos que analisam a relação entre as práticas de contabilidade de gestão ambiental e a inovação ainda apresenta lacunas na literatura.

Segundo Ferreira, Moulang e Hendro (2010), presume-se que a inovação é importante para a maioria das organizações. No entanto,

[...] embora os benefícios decorrentes de inovações possam ser documentados, o papel dos sistemas de contabilidade relacionados à sustentabilidade (como o EMA) como um driver para inovações dentro das organizações, permanece largamente inexplorado. (FERREIRA; MOULANG; HENDRO, 2010, p. 921)

Com esse foco, os autores investigaram, em 40 grandes empresas australianas, se o uso do sistema EMA pode levar a inovações nas empresas, bem como qual o papel da estratégia em relação ao uso do sistema EMA e inovação. Nem todas as hipóteses testadas foram confirmadas, o que sugere a existência de divergências na literatura sobre essa temática, razão pela qual se torna oportuno a realização de nova investigação.

Diante do exposto, este estudo busca responder à seguinte pergunta de pesquisa: Qual é a relação das práticas de contabilidade de gestão ambiental com inovação em empresas industriais de grande porte estabelecidas no Estado do Rio Grande do Sul, Brasil? O estudo objetiva identificar a relação entre práticas de contabilidade de gestão ambiental $e$ inovação, fundamentado no modelo teórico de Ferreira, Moulang e Hendro (2010), em empresas industriais de grande porte estabelecidas no Rio Grande do Sul, Brasil. Adicionalmente, pretende-se identificar as práticas de EMA utilizadas nessas empresas e os benefícios percebidos pelos participantes da pesquisa na utilização do sistema EMA.

O estudo justifica-se por contribuir para o entendimento da influência das práticas de contabilidade de gestão ambiental nos processos de inovação das empresas. Também por contribuir para o entendimento das práticas de EMA utilizadas por grandes corporações estabelecidas no Brasil. Busca ainda fornecer evidências dos benefícios percebidos por essas empresas na utilização do sistema EMA. Ao replicar o instrumento de pesquisa de Ferreira, Moulang e Hendro (2010) e utilizar o constructo teórico $e$ as hipóteses desenvolvidas pelos autores, pretende-se contribuir para a consolidação do tema na literatura pertinente, visto que nem todas as hipóteses estabelecidas pelos autores foram confirmadas em sua pesquisa, o que abre perspectiva para a realização de novos estudos sobre esta temática.

\section{Significado de Environmental Management Accounting (EMA)}

Uma preocupação emergente nas organizações está relacionada à identificação de alternativas que promovam a melhoria dos processos de gestão ambiental. O desenvolvimento de sistemas específicos de gestão ambiental tem auxiliado os gestores de diversas formas nesse processo (SCHALTEGGER; BURRITT, 2000; BURRITT, 2004; DE BEER; FRIEND, 2006; FERREIRA; MOULANG; HENDRO, 2010). De acordo 
com Masanet-Llodra (2006, p. 393), para a Comunidade Europeia, o sistema de gestão ambiental deve ser compreendido como "[...] parte do sistema de gestão que inclui a estrutura organizacional, as responsabilidades, a prática, os procedimentos, os processos e os recursos para determinar e empreender uma política ambiental rigorosa na empresa".

Na contabilidade, a área que trata especificamente dessa temática é a contabilidade ambiental, que envolve processos relacionados à identificação, coleta, análise e utilização de uma gama de informações de atividades relacionadas à gestão ambiental das empresas, que servem de suporte ao processo decisório das organizações (SCHALTEGGER; BURRITT, 2000; BURRITT, 2004; BURRITT; MASANET-LLODRA, 2006; SAKA, 2006; STANISKIS; STASISKIENE, 2006; FERREIRA; MOULANG; HENDRO, 2010). A contabilidade ambiental auxilia na expressão de passivos ambientais e sociais, como nos custos ambientais. (DE BEER; FRIEND, 2006)

Segundo Burrit, Hahn e Schaltegger (2002), a contabilidade ambiental pode ser dividida em duas partes. A primeira, denominada Contabilidade de Gestão Ambiental (Environmental Management Accounting - EMA), está relacionada às técnicas e aos procedimentos utilizados pelos profissionais da contabilidade, na obtenção de informações, de diferentes naturezas (física e monetária) que suportam o processo decisório dos gestores, tanto nas questões relacionadas ao processo de gestão ambiental das organizações, como na análise do impacto das operações das empresas com o meio ambiente. A segunda, denominada de Contabilidade Financeira Ambiental (Environmental Financial Accounting - EFA), está relacionada aos registros contábeis que apuram os resultados das operações da empresa, em determinado período.

O foco do sistema EMA é mais amplo e, dessa forma, tem ocupado maior espaço no processo decisório das organizações. A estruturação de um sistema de contabilidade de gestão ambiental, sob a perspectiva gerencial, enfatiza as necessidades do processo decisório interno da organização, por isso possui maior abrangência (SCHALTEGGER; BURRITT, 2000). O departamento de contabilidade das organizações vem sendo reformulado a fim de atender esta demanda, passando a utilizar sistema de contabilidade de gestão ambiental (EMA), como parte da infraestrutura contábil que fornece informações aos gestores. (GALE, 2006, SCAVONE, 2006; SCHALTEGGER et al., 2009)

Especificamente em relação a esse sistema, Bennett, Bouma e Wolters (2002, p. 1) explicam que a Contabilidade de Gestão Ambiental (EMA) compreende "[...] a geração, análise e uso de informações financeiras e não-financeiras, a fim de otimizar o desempenho ambiental e econômico das empresas, e para conseguir negócios sustentáveis". Portanto, envolve desde processos de

[...] identificação, coleta, cálculo (estimativa), análise e elaboração de relatórios internos, com informações sobre uso de materiais, energia e custos ambientais, bem como de outros dados $e$ informações que possam contribuir para a tomada de decisões, capazes de contribuir para a proteção do ambiente. (VASILE; MAN, 2012, p. 566)

\section{Benefícios do Uso da Environmental Management Accounting (EMA)}

O sistema de Contabilidade de Gestão Ambiental (EMA) é um instrumento de gestão relativamente novo, desenvolvido com o propósito inicial de rastrear e controlar custos ambientais e fluxos físicos ambientais (BURRITT; SAKA, 2006). Porém, com seus potenciais benefícios, a sua utilização tem se expandido ao longo dos anos, contribuindo para a melhoria dos processos decisórios. (SCHALTEGGER; BURRITT, 2000; BURRITT, 2004; DE BEER; FRIEND, 2006; FERREIRA; MOULANG; HENDRO, 2010; CHRIST; BURRITT, 2013)

De acordo com Ferreira, Moulang e Hendro (2010), com a utilização do sistema EMA, tornou-se possível a obtenção de uma série de benefícios econômicos, além da melhoria do desempenho ambiental das organizações. Isso é possível devido ao aumento dos diferentes tipos de informações, físicas e monetárias, que qualificam o processo decisório dos gestores. (SCHALTEGGER; BURRITT, 2000; BURRITT; HAHN; SCHALTEGGER, 2002; BENNETT; RIKHARDSSON; SCHALTEGGER, 2003; BURRITT, 2004; DE BEER; FRIEND, 2006) 
Entre os principais benefícios associados ao uso do sistema EMA, identificados na literatura contábil, destacam-se: a identificação de oportunidades de redução de custos, o estabelecimento de um mix de produtos que melhora as decisões de preços, o desenvolvimento de ações que procuram evitar custos futuros associados com decisões de investimento, o desenvolvimento de processos mais eficientes, a melhoria da reputação organizacional, a atração de recursos humanos, e a melhoria do desempenho ambiental. (FROST; WILMSHURST, 2000; BURRITT; HAHN; SCHALTEGGER, 2002; BENNETT; RIKHARDSSON; SCHALTEGGER, 2003; DEEGAN, 2003; BURRITT, 2004; HANSEN; MOWEN, 2005; DE BEER; FRIEND, 2006; GALE, 2006; SCAVONE, 2006; FERREIRA; MOULANG; HENDRO, 2010; CHRIST; BURRITT, 2013)

No Brasil, a temática ainda é pouco investigada, mas estudos desenvolvidos com essa abordagem teórica sugerem alguns benefícios às organizações, obtidos a partir da utilização deste sistema. Segundo Vellani e Ribeiro (2009, p. 39), a contabilidade de gestão ambiental pode categorizar as ações ecológicas empresariais e possibilitar o fornecimento de informações sobre os eventos relacionados com a ecoeficiência do negócio, melhorando os processos de gestão da empresa.

Nunes, Pfitscher e Uhlmann (2011) e Pamplona et al. (2011) explicam que a utilização do sistema de contabilidade de gestão ambiental pode aprimorar os processos de gestão ambiental das organizações. Em outra perspectiva, Braz et al. (2009, p. 79) procuraram desenvolver uma metodologia para "[...] reconhecer, mensurar e evidenciar o resultado social e ambiental das ações decorrentes das atividades de uma autarquia municipal do interior do Estado de São Paulo". Com a aplicação da proposta desenvolvida pelos autores, obtiveram "[...] redução de materiais descartados e menor extração de matéria-prima da natureza". (BRAZ et al., 2009, p. 79)

De maneira geral, a literatura contábil relacionada a essa área tem sugerido que os benefícios advindos da utilização do sistema EMA não se restringem apenas a fatores econômico-financeiros (BURRITT; SAKA, 2006; FERREIRA; MOULANG; HENDRO, 2010; CHRIST; BURRITT, 2013). A melhoria nos processos de gestão pode proporcionar outros benefícios, que auxiliam na eficiência do negócio. Processos eficazes auxiliam na customização dos recursos consumidos pela empre- sa, maximizando seus resultados. (SCHALTEGGER; BURRITT, 2000; BURRITT, 2004; HANSEN; MOWEN, 2005; DE BEER; FRIEND, 2006; VELLANI; RIBEIRO, 2009; FERREIRA; MOULANG; HENDRO, 2010; NUNES; PFITSCHER; UHLMANN, 2011; PAMPLONA et al., 2011; CHRIST; BURRITT, 2013)

Depreende-se do exposto que a adoção do sistema de contabilidade de gestão ambiental pode proporcionar vantagem competitiva às empresas e, por consequência, agregar valor à organização. Assim sendo, espera-se que cada vez mais organizações desenvolvam tal sistema, com vistas a qualificar seu processo de gestão ambiental. Apesar dos benefícios que podem ser obtidos com o sistema de contabilidade de gestão ambiental, observa-se que a utilização desse sistema ainda não está totalmente disseminada entre as empresas, o que abre perspectivas para a realização de novos estudos.

Evidências recentes tem sugerido que isso possivelmente pode estar relacionado com a falta de conhecimento dos profissionais que atuam nessa área, o que pode decorrer da falta de oferta de disciplinas específicas enfatizando a contabilidade e a gestão ambiental nas matrizes curriculares dos cursos de graduação em Ciências Contábeis oferecidos pelas instituições de ensino superior (MARTENDAL et al., 2013). Nesse sentido, torna-se necessário inicialmente a formação e a capacitação de profissionais docentes, que possam contribuir na disseminação do conhecimento sobre tal área, bem como o ajuste das propostas pedagógicas dos referidos cursos que introduzam tal abordagem na formação acadêmica.

Da mesma forma, é necessário que os gestores das organizações compreendam a importância do estabelecimento de práticas de gestão ambiental e percebam os potenciais benefícios que podem ser obtidos com a implementação de tal sistema de gestão. Isso pode, por exemplo, contribuir para o desenvolvimento de uma consciência crítica dentro da organização, o que é importante ao propósito de desenvolver uma cultura de gestão ambiental nas empresas. (BARBIERI, 2006; MARTENDAL et al., 2013)

Sob esse aspecto, Christ e Burritt (2013) explicam que apenas as organizações maiores, ambientalmente sensíveis e com estratégia ambiental bem definida são mais propensas à implementação do sistema EMA. Logo, é oportuno investigar em empresas de grande 
porte estabelecidas no Brasil sobre o uso do sistema de contabilidade de gestão ambiental, as principais práticas de EMA adotadas por essas empresas e os principais benefícios obtidos por essas organizações com a sua utilização, motivações que estimulam esta investigação.

\section{Modelo Teórico de Análise}

As melhorias nos processos de gestão ambiental, alcançadas a partir da utilização da contabilidade de gestão ambiental, representam uma inovação nas empresas. Assim, é possível admitir que a contabilidade atua como um driver para inovações nas organizações. No entanto, é preciso considerar os efeitos das variáveis contingenciais sobre a utilização de tal sistema. Para examinar essas relações em empresas industriais de grande porte estabelecidas no Brasil, procurou-se utilizar o modelo teórico de análise desenvolvido por Ferreira, Moulang e Hendro (2010), apresentado na Figura 1.

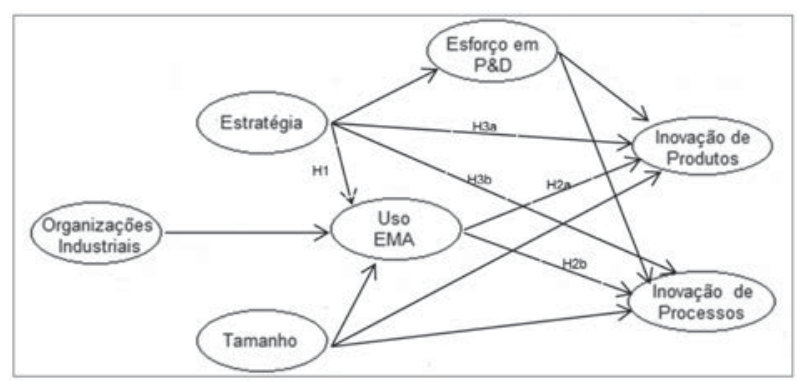

Figura 1: Modelo teórico de análise das relações entre EMA e Inovação

Fonte: Adaptada de Ferreira, Moulang e Hendro (2010)

Observa-se, na Figura 1, que as proposições teóricas elaboradas pelos autores levam em consideração a influência de dois fatores contingenciais sobre a utilização do sistema de contabilidade de gestão ambiental: a estratégia; e o tamanho da organização. Segundo os autores, tais fatores podem influenciar a utilização ou não desse sistema. A estratégia utilizada pela empresa pode influenciar a quantidade de recursos investidos em pesquisa e desenvolvimento ( $\mathrm{P} \& \mathrm{D})$, que também pode influenciar os níveis de inovação da empresa. A partir das relações dos constructos teóricos identificados na pesquisa, os autores estabeleceram cinco hipóteses, que foram testadas empiricamente, apresentadas no Quadro 1.

\begin{tabular}{|c|c|}
\hline HiPótESES INVESTIGADAS NA PESQUISA & RESULTADOS \\
\hline $\begin{array}{c}\text { H1: Há uma associação positiva entre } \\
\text { a estratégia prospectora e uso EMA. }\end{array}$ & Não suportada \\
\hline $\begin{array}{c}\text { H2a: Há uma associação positiva entre } \\
\text { o uso EMA e inovação de produtos. }\end{array}$ & Não suportada \\
\hline $\begin{array}{c}\text { H2b: Há uma associação positiva entre } \\
\text { o uso EMA e inovação de processos. }\end{array}$ & Suportada \\
\hline $\begin{array}{c}\text { H3a: Há uma associação positiva } \\
\text { entre a estratégia prospectora e } \\
\text { inovação dos produtos. }\end{array}$ & Não suportada \\
\hline $\begin{array}{c}\text { H3b: Há uma associação positiva } \\
\text { entre a estratégia prospectora e } \\
\text { inovação dos processos. }\end{array}$ & Não suportada \\
\hline
\end{tabular}

Quadro 1: Hipóteses de pesquisa do modelo teórico de análise das relações entre EMA e Inovação

Fonte: Adaptado de Ferreira, Moulang e Hendro (2010)

A hipótese $\mathrm{H} 1$ estabelece que existe associação positiva entre a estratégia estabelecida pelas organizações, nesse caso, a estratégia prospectora, e o uso do sistema EMA. Organizações que perseguem uma estratégia prospectora, entendem e enfatizam a importância do desenvolvimento de produtos inovadores (MILES; SNOW, 1978). De acordo com Gimenez et al. (1999, p. 59), a estratégia prospectora é "[...] caracterizada por elevada busca de mercados e inovação de produtos e processos". Gosselin (1997) explica que a estratégia prospectora está associada com a adoção do gerenciamento de atividades. Apesar dos apontamentos identificados na literatura anterior, os quais sugerem tal relação, no estudo realizado por Ferreira, Moulang e Hendro (2010), essa associação não foi confirmada.

As hipóteses $\mathrm{H} 2 \mathrm{a}$ e $\mathrm{H} 2 \mathrm{~b}$ propõem que há associação positiva entre uso do sistema de contabilidade de gestão ambiental e inovação de produtos e de processos, respectivamente. Evidências encontradas na literatura têm sugerido que as organizações desenvolvem sistemas de controle gerencial, com o propósito de manter ou de aumentar sua vantagem competitiva. Ao inovar em processos e produtos, as empresas procuram aumentar sua rentabilidade (DRAKE; HAKA; RAVENSCROFT, 1999; DAVILA, 2000; ADAMS; ZUTSHI, 2004; DAVILA; FOSTER; LI, 2009; REVELLINO; MOURITSEN, 2009; FERREIRA; MOULANG; HENDRO, 2010; OYADOMARI et al., 2010; NISIYAMA; OYADOMARI, 2012). No entanto, os resultados encontrados por Ferreira, Moulang e Hendro 
(2010) suportaram parcialmente as hipóteses testadas, já que encontraram associação positiva apenas entre uso do sistema EMA e inovação de processos.

As hipóteses $\mathrm{H} 3 \mathrm{a}$ e $\mathrm{H} 3 \mathrm{~b}$ investigam a relação entre estratégia prospectora e inovação de processos e produtos. Uma empresa que desenvolve a estratégia prospectora tenta ser a primeira no mercado, destacando-se pela inovação e flexibilidade para responder rapidamente às mudanças nas condições de mercado (FERREIRA; MOULANG; HENDRO, 2010). Segundo Milles e Snow (1978), organizações que adotam estratégia prospectora são bem-sucedidas. Hambrick (1983) explica que as empresas prospectoras tendem a prosperar em ambientes dinâmicos e inovadores, pelo aproveitamento de oportunidades de crescimento. Portanto, quanto maior for à ênfase da organização em ser a primeira no mercado, maior tende a ser o nível de inovação de produtos (MILLES; SNOW, 1978). Apesar de tais evidências, ambas as hipóteses não foram confirmadas no estudo de Ferreira, Moulang e Hendro (2010).

Nesse caso, as evidências apresentadas por Ferreira, Moulang e Hendro (2010), a partir da análise das relações apresentadas na Figura 1, indicam que, em empresas industriais que utilizam sistema de contabilidade de gestão ambiental, houve melhoria nos processos de gestão ambiental, o que caracteriza a inovação de processos. Em vista das divergências encontradas na literatura, torna-se oportuno a realização deste estudo em outro ambiente, no caso, testar tais relacionamentos em empresas de grande porte estabelecidas no Brasil.

\section{Método e Procedimentos da Pesquisa}

Com o propósito de se identificar a relação existente entre práticas de contabilidade de gestão ambiental e inovação, a partir do modelo teórico de Ferreira, Moulang e Hendro (2010), realizou-se pesquisa descritiva conforme preceitos de Gil (2002), por meio de levantamento, com abordagem quantitativa dos dados. O estudo de Ferreira, Moulang e Hendro (2010) é replicado em empresas de grande porte, estabelecidas no Estado do Rio Grande do Sul, Brasil, com o argumento de que nem todas as hipóteses elaboradas pelos autores foram confirmadas, o que sugere a existência de lacunas teóricas e empíricas que justificam a sua reprodução em outro ambiente.
A população objeto de estudo foi selecionada de maneira intencional, alcançada por acessibilidade, por isso centrou-se no Estado do Rio Grande do Sul, Brasil. Como critério de seleção das empresas participantes da pesquisa estabeleceu-se que elas deveriam ser empresas industriais, de grande porte, localizadas no Estado do Rio Grande do Sul, Brasil. O critério de seleção do porte das empresas foi estabelecido a partir da receita operacional bruta. De acordo com o Banco Nacional de Desenvolvimento Econômico e Social (BNDES), são consideradas empresas de grande porte aquelas com faturamento anual superior a $\mathrm{R} \$ 300.000 .000,00$ (trezentos milhões de reais).

A escolha por empresas industriais de grande porte deve-se ao fato de que as suas atividades operacionais tendem a impactar mais fortemente de modo negativo o meio ambiente. Essas empresas consomem recursos naturais como matéria-prima principal para o desenvolvimento de seus produtos. Logo, estão mais propensas ao desenvolvimento de sistemas de contabilidade de gestão ambiental, como o sistema EMA, para a melhoria dos processos de gestão (FERREIRA; MOULANG; HENDRO, 2010). A coleta de dados ocorreu entre os meses de novembro e dezembro de 2012 e alcançou 21 empresas industriais. Os respondentes da pesquisa foram os gestores do departamento de contabilidade.

O instrumento de pesquisa utilizado para a coleta de dados consistiu do questionário desenvolvido por Ferreira, Moulang e Hendro (2010). O questionário é composto por 37 questões objetivas, de múltipla escolha, dividido em quatro partes. A primeira busca identificar o uso do sistema EMA pelas empresas pesquisadas e as principais práticas de contabilidade de gestão ambiental que adotam. A segunda está relacionada à identificação da estratégia de gestão ambiental utilizada pela empresa. A terceira parte busca investigar as questões relacionadas à inovação de processos e produtos. A última parte diz respeito aos benefícios percebidos pelos gestores, a partir da utilização do sistema EMA.

Inicialmente procedeu-se à tradução do instrumento de pesquisa, que em seguida foi analisado por dois pesquisadores e respondido a fim de se realizar um pré-teste. Da mesma forma foi realizado um pré-teste com um profissional da área contábil que atua em uma empresa industrial para identificar se os termos utilizados no instrumento de pesquisa traduzido estão em 
conformidade com a linguagem profissional utilizada na área contábil. Efetuado o pré-teste, procedeu-se a coleta de dados.

Os dados coletados foram tabulados em planilhas eletrônicas e posteriormente importados aos softwares Statistical Package for the Social Sciences (SPSS) e Partial Least Squares (PLS), para o tratamento estatístico. As técnicas de análise estatística utilizadas na pesquisa foram: estatística descritiva, matriz de correlação $e$ modelagem por equações estruturais (SEM), com uso do método dos mínimos quadrados parciais PLS.

O teste das hipóteses de pesquisa (Quadro 1) foi estruturado conforme a Figura 1, com base no modelo de equações estruturais (SEM) proposto por Ferreira, Moulang e Hendro (2010). A modelagem por equações estruturais PLS é uma técnica utilizada para a análise dos relacionamentos entre variáveis em pequenas amostras. Esta técnica apresenta a característica de estimação de múltiplas relações e inter-relacionamentos em investigações de relações de dependência (HAIR JR. et al., 1998). Os resultados da pesquisa são apresentados a seguir.

\section{AnÁLISE E INTERPRETAÇÃo dos Resultados}

Nesta seção apresenta-se a análise e a interpretação dos resultados da pesquisa. Inicialmente estabeleceu-se a caracterização em relação ao tipo de empresas industriais que participaram do estudo. A Tabela 1 apresenta uma síntese dos resultados.
Tabela 1: Tipos de empresas industriais participantes da pesquisa

\begin{tabular}{|c|c|c|}
\hline Tipo de empresa industrial & $\begin{array}{c}\text { Freq. } \\
\text { Absoluta }\end{array}$ & Freq. Relativa \\
\hline Beneficiamento de tabaco & 7 & $33,33 \%$ \\
\hline Metalurgia & 7 & $33,33 \%$ \\
\hline Embalagens plásticas & 3 & $14,29 \%$ \\
\hline Fabricação de alimentos & 3 & $14,29 \%$ \\
\hline Beneficiamento de borracha & 1 & $4,76 \%$ \\
\hline Total & $\mathbf{2 1}$ & $\mathbf{1 0 0 , 0 0 \%}$ \\
\hline
\end{tabular}

Fonte: Dados da pesquisa

É possível observar, na Tabela 1, que participaram da pesquisa 21 empresas industriais de grande porte, que se encontram localizadas no Estado do Rio Grande do Sul, Brasil. A maioria das empresas participantes da pesquisa $(66,66 \%)$ são indústrias de beneficiamento de tabaco (7) e metalurgia (7). As demais $(33,34 \%)$ atuam nos segmentos de fabricação de embalagens plásticas (3), alimentos (3) e beneficiamento de borracha (1).

Realizada a identificação do tipo de empresa industrial participante da pesquisa, procedeu-se à análise das práticas de contabilidade de gestão ambiental, utilizadas por essas empresas, e as práticas de inovação de processos e produtos desenvolvidos nos últimos três anos. Para a análise desses resultados, optou-se pela aplicação da estatística descritiva de média e desvio padrão dos indicadores dos constructos teóricos analisados no estudo, e suas respectivas cargas fatoriais. Os resultados desta análise são apresentados na Tabela 2 .

Tabela 2: Cargas fatoriais dos constructos do modelo de avaliação PLS

\begin{tabular}{|c|c|c|c|c|c|c|}
\hline Constructos Analisados & \multicolumn{2}{|c|}{ INTERVALO } & \multicolumn{2}{|c|}{ Est. Descritiva } & \multicolumn{2}{|c|}{ SEM PLS } \\
\hline Environmental Management Accounting (EMA) & Teórico & Real & Média & $\begin{array}{l}\text { Desvio } \\
\text { Padrão }\end{array}$ & Carga & $p$-value \\
\hline Identificação de custos relacionados ao meio ambiente. & $0-6$ & $2-6$ & 4,19 & 1,21 & 0,863 & 0,0000 \\
\hline Estimativa de obrigações contingentes relacionadas ao meio.ambiente. & $0-6$ & $0-6$ & 3,19 & 2,02 & 0,846 & 0,0000 \\
\hline Classificação de custos relacionados ao meio ambiente. & $0-6$ & $1-6$ & 3,57 & 1,69 & 0,804 & 0,0000 \\
\hline $\begin{array}{l}\text { Alocação de custos relacionados ao meio am- } \\
\text { biente para processos de produção. }\end{array}$ & $0-6$ & $0-6$ & 4,24 & 1,70 & 0,904 & 0,0000 \\
\hline Alocação de custos relacionados ao meio ambiente para produtos. & $0-6$ & $0-6$ & 4,05 & 1,80 & 0,824 & 0,0000 \\
\hline $\begin{array}{l}\text { Introdução ou melhoria de gerenciamento de cus- } \\
\text { tos relacionados ao meio ambiente. }\end{array}$ & $0-6$ & $0-6$ & 4,24 & 1,55 & 0,930 & 0,0000 \\
\hline Criação e uso de contas de custos relacionados ao meio ambiente. & $0-6$ & $0-6$ & 3,33 & 1,93 & 0,832 & 0,0000 \\
\hline
\end{tabular}




\begin{tabular}{|c|c|c|c|c|c|c|}
\hline $\begin{array}{l}\text { Desenvolvimento e uso de indicadores de desem- } \\
\text { penho relacionados ao meio ambiente. }\end{array}$ & $0-6$ & $0-6$ & 4,10 & 2,34 & 0,767 & 0,0000 \\
\hline Avaliações de custo do ciclo de vida de produtos. & $0-6$ & $0-6$ & 3,95 & 1,88 & 0,834 & 0,0000 \\
\hline $\begin{array}{c}\text { Análise de estoque de produtos (ex.: especificação dos ti- } \\
\text { pos e quantidades de materiais e energia requeridos e a } \\
\text { quantidade de resíduos liberados no meio ambiente). }\end{array}$ & $0-6$ & $0-6$ & 4,14 & 1,90 & 0,940 & 0,0000 \\
\hline $\begin{array}{l}\text { Análise de impacto de produto (ex: avaliação do efei- } \\
\text { to ambiental de projetos de produtos concorrentes) }\end{array}$ & $0-6$ & $0-6$ & 3,57 & 2,09 & 0,813 & 0,0000 \\
\hline $\begin{array}{l}\text { Análise de melhoria de produto (ex.: identificação de opor- } \\
\text { tunidades de redução de impacto ambiental). }\end{array}$ & $0-6$ & $0-6$ & 4,05 & 1,53 & 0,836 & 0,0000 \\
\hline Inovação de Produtos & Teórico & Real & Média & $\begin{array}{l}\text { Desvio } \\
\text { Padrão }\end{array}$ & Carga & $p$-value \\
\hline Durante os últimos três anos nós lançamos poucos/muitos produtos novos & $1-7$ & $1-6$ & 3,76 & 1,84 & 0,928 & 0,0000 \\
\hline $\begin{array}{l}\text { Durante os últimos três anos nós lançamos poucas/mui- } \\
\text { tas modificações para produtos que já existiam. }\end{array}$ & $1-7$ & $1-6$ & 4,10 & 1,34 & 0,878 & 0,0000 \\
\hline $\begin{array}{l}\text { Com respeito a novos produtos, nós somos raramente/ } \\
\text { muito frequentemente os primeiros no mercado. }\end{array}$ & $1-7$ & $1-7$ & 3,76 & 1,87 & 0,940 & 0,0000 \\
\hline $\begin{array}{l}\text { A porcentagem de novos produtos em nosso portfólio de pro- } \\
\text { dutos é muito menor/maior que a média da indústria. }\end{array}$ & $1-7$ & $1-7$ & 3,95 & 1,20 & 0,833 & 0,0000 \\
\hline Inovação de Processos & Teórico & Real & Média & $\begin{array}{l}\text { Desvio } \\
\text { Padrão }\end{array}$ & Carga & $p$-value \\
\hline $\begin{array}{l}\text { Durante os três últimos anos nós introduzimos pou- } \\
\text { cos/muitos novos processos de produção. }\end{array}$ & $1-7$ & $1-6$ & 4,33 & 1,39 & 0,940 & 0,0000 \\
\hline $\begin{array}{l}\text { Durante os três últimos anos nós introduzimos poucas/ } \\
\text { muitas modificações nos processos de produção. }\end{array}$ & $1-7$ & $2-6$ & 5,05 & 1,20 & 0,926 & 0,0000 \\
\hline $\begin{array}{l}\text { Com relação aos processos novos de produção, nós somos mui- } \\
\text { to raramente/muito frequentemente os primeiros a introduzi-los. }\end{array}$ & $1-7$ & $1-7$ & 4,81 & 1,60 & 0,787 & 0,0000 \\
\hline $\begin{array}{l}\text { A frequência de melhorias nos processos de produção de nos- } \\
\text { sa empresa é muito menor/maior que a média da indústria. }\end{array}$ & $1-7$ & $2-7$ & 4,90 & 1,26 & 0,772 & 0,0001 \\
\hline
\end{tabular}

Fonte: Dados da pesquisa

Verifica-se, na Tabela 2, que nem todas as empresas industriais de grande porte participantes da pesquisa adotam práticas de contabilidade de gestão ambiental. Apesar da convergência encontrada na literatura de que a utilização do sistema de contabilidade de gestão ambiental pode contribuir para a melhoria do processo decisório das organizações (FROST; WILMSHURST, 2000; BURRITT; HAHN; SCHALTEGGER, 2002; BENNETT; RIKHARDSSON; SCHALTEGGER, 2003; DEEGAN, 2003; BURRITT, 2004; HANSEN; MOWEN, 2005; DE BEER; FRIEND, 2006; GALE, 2006; SCAVONE, 2006; FERREIRA; MOULANG; HENDRO, 2010; CHRIST; BURRITT, 2013), observa-se que algumas empresas ainda não estão sensíveis a tais questões. A não utilização de tais informações afeta a qualidade do processo decisório dessas empresas, o que pode influenciar em perda de competitividade e afetar o seu desempenho. (FERREIRA; MOULANG; HENDRO, 2010; CHRIST; BURRITT, 2013)

Dentre as que utilizam tais sistemas de gestão, as principais práticas utilizadas estão relacionadas com: alocação de custos relacionados ao meio ambiente para processos de produção $(4,24)$; introdução ou melhoria de gerenciamento de custos relacionados ao meio ambiente $(4,24)$; identificação de custos relacionados ao meio ambiente $(4,19)$; análise de estoque de produtos (ex.: especificação dos tipos e quantidades de materiais e energia requeridos e a quantidade de resíduos liberados no meio ambiente) $(4,14)$; desenvolvimento e uso de indicadores de desempenho relacionados ao meio ambiente $(4,10)$; alocação de custos relacionados ao meio ambiente para produtos (4,05); e, análise de 
melhorias de produtos (ex.: identificação de oportunidades de redução de impacto ambiental).

Essas práticas, convergentes com o observado na literatura, denotam que a utilização de tais sistemas de gestão contribuem para a identificação mais adequada de um número maior de informações financeiras e não financeiras relacionadas ao meio ambiente e as atividades da empresa, o que qualifica o processo decisório dos gestores dessas empresas. (FROST; WILMSHURST, 2000; GALE, 2006; SCAVONE, 2006)

No que se refere às ações desenvolvidas pelas empresas pesquisadas, relacionadas à inovação de processos e produtos, observa-se que todas as empresas, de alguma forma, desenvolveram ou iniciaram alguma atividade inovativa, nos últimos três anos. Especificamente em relação à inovação de produtos, a maioria das empresas pesquisadas informou que, durante os últimos três anos, ocorreu o lançamento de muitas modificações para produtos que já existiam $(4,10)$. Da mesma forma, a maioria das empresas pesquisadas destacou que a porcentagem de novos produtos em seu portfólio de produtos é muito maior que a média da indústria $(3,95)$.

Em se tratando da inovação de processos, nota-se que as modificações implementadas pelas empresas em seus processos de produção foram ainda maiores. As médias encontradas nas afirmativas relacionadas à ocorrência de muitas modificações nos processos de produção neste período $(5,05)$, bem como a frequência de melhorias realizadas nos processos de produção dessas empresas, comparativamente a média das indústrias que atuam no mesmo setor $(4,90)$, foram maiores que as médias encontradas para a inovação de produtos. Os resultados encontrados mostram que parte das empresas pesquisadas estão focalizadas em melhorar continuamente os processos de produção, o que pode contribuir para a melhoria do desempenho econômico, financeiro e ambiental dessas empresas.

Em relação à análise fatorial exploratória dos indicadores de cada constructo investigado na pesquisa, observa-se que todos os indicadores carregados alcançaram um índice de confiabilidade superior a 0,70. De acordo com Hair Jr. (1998, p. 90), o Alfa de Cronbach é uma medida comumente usada para auferir a confiabilidade de um conjunto de dados: "[...] os valores variam de 0 a 1, com as medidas mais altas indicando maior confiabilidade entre os indicadores, sendo os valores de 0,60 a 0,70 considerados o limite inferior de aceitabilidade". Dessa forma, nenhum indicador observado no estudo foi excluído.

$\mathrm{Na}$ etapa seguinte da pesquisa, realizou-se uma síntese dos principais benefícios destacados pelas empresas pesquisadas, a partir da utilização do sistema de contabilidade de gestão ambiental. O Gráfico 1 apresenta os resultados dessa análise.

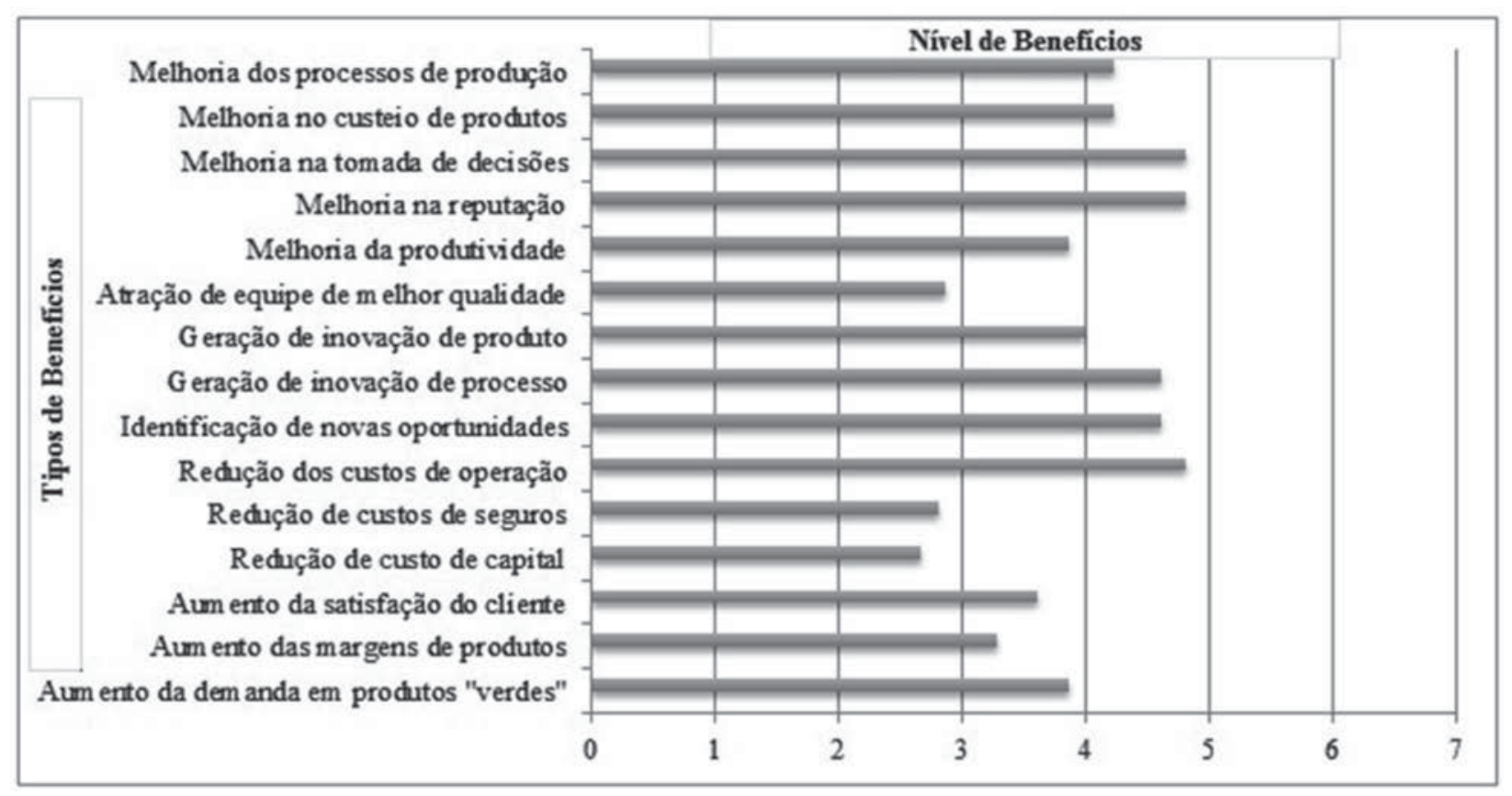

Gráfico 1: Benefícios percebidos a partir da utilização da EMA

Fonte: Dados da pesquisa 
Observa-se no Gráfico 1 que são vários os benefícios percebidos pelos gestores participantes da pesquisa, em relação à utilização de sistemas específicos de contabilidade de gestão ambiental e à adoção de práticas de contabilidade de gestão ambiental por parte dessas empresas. Da mesma forma, como no estudo realizado por Ferreira, Moulang e Hendro (2010), foram realizados 15 questionamentos aos gestores para identificar em uma escala de 0 a 7, os níveis de benefícios percebidos, a partir da utilização do sistema EMA.

Os resultados mostram que os principais benefícios percebidos por essas empresas estão relacionados com: redução dos custos de operação $(4,81)$; melhoria na reputação da empresa $(4,81)$; melhoria na tomada de decisões $(4,81)$; identificação de novas oportunidades de negócio $(4,62)$; geração de inovação de processos $(4,62)$; melhoria dos processos de produção $(4,24)$; e, melhoria no custeio de produtos $(4,24)$. Por outro lado, se observa nos resultados deste estudo, que são baixos os níveis de benefícios percebidos pelos gestores em relação a: atração de equipe de trabalho de melhor qualidade $(2,86)$; redução de custos de seguros $(2,81)$; e, redução do custo de capital $(2,67)$. Tais resultados sugerem que estes benefícios podem não ser determinantes para a utilização do sistema EMA nas empresas industriais de grande porte pesquisadas, $\mathrm{O}$ que motiva a realização de novos estudos.

Os resultados encontrados evidenciam que os benefícios obtidos pelas empresas que utilizam o sistema EMA não são apenas econômico-financeiros. A melhoria nos processos de gestão tende a proporcionar benefícios que auxiliam na eficiência das atividades da empresa, o que contribui para o melhor aproveitamento dos recursos disponíveis na organização, e, por consequência, na maximização de seus resultados. Assim, espera-se que empresas industriais que exploram recursos extraídos do meio ambiente, utilizem sistemas de contabilidade de gestão ambiental, visto que seus potenciais benefícios agregam valor à organização.

Identificadas as práticas de EMA adotadas nas empresas pesquisadas, bem como os benefícios percebidos com a sua utilização, na sequência, analisaram-se as relações dos constructos teóricos elaborados para a realização deste estudo, a partir da modelagem por equações estruturais PLS. Inicialmente realizaram-se os testes estatísticos de análise do modelo (Tabela 3) e, em seguida, a análise da existência de correlação entre os constructos investigados na pesquisa (Tabela 4).

Tabela 3: Testes estatísticos do modelo

\begin{tabular}{|c|c|c|c|c|}
\hline Variável Latente & $\begin{array}{c}\text { Coeficiente } \\
\text { R-squared }\end{array}$ & $\begin{array}{c}\text { Coeficiente de } \\
\text { confiabilidade } \\
\text { composta }\end{array}$ & $\begin{array}{c}\text { Coeficiente Alfa } \\
\text { Cronbach }\end{array}$ & $\begin{array}{c}\text { Variância } \\
\text { média explicada } \\
\text { (VME) }\end{array}$ \\
\hline Organ. Industrial & 0,0000 & 1,0000 & 1,0000 & 1,0000 \\
\hline Estratégia & 0,0000 & 0,9200 & 0,8743 & 0,7944 \\
\hline Tamanho & 0,0000 & 0,9272 & 0,8610 & 0,8646 \\
\hline Uso EMA & 0,6162 & 0,9691 & 0,9664 & 0,7241 \\
\hline Esforço em P\&D & 0,0740 & 1,0000 & 1,0000 & 1,0000 \\
\hline Inovação de Produtos & 0,8075 & 0,9419 & 0,9172 & 0,8024 \\
\hline Inovação de Processos & 0,4583 & 0,9182 & 0,8866 & 0,7388 \\
\hline
\end{tabular}

Fonte: Dados da pesquisa

Nota-se na Tabela 3 que, de maneira geral, os constructos analisados alcançaram a confiabilidade desejada. Desta forma, todos os indicadores foram incluídos no modelo. Os índices do Coeficiente Alfa Cronbach encontrados na pesquisa sugerem a validade das variáveis indicadoras em relação às suas variáveis latentes, razão pela qual todas elas foram aceitas como integrantes dos constructos teóricos investigados no estudo.

Observa-se ainda, na Tabela 3, que a variância média explicada (VME) e o índice de confiabilidade composta também alcançaram índices superiores a 0,5, mínimo aceitável para sua validação (HAIR Jr. et al., 1998). A raiz quadrada da variância média extraída 
para os constructos analisados foi superior às correlações entre as variáveis latentes (Tabela 4). Denota-se, assim, a existência de validade discriminante entre as variáveis latentes investigadas na pesquisa. (FORNELL; LARCKER, 1981; BIDO et al., 2009; BIDO et al., 2010; MENEZES; GUIMARÃES; BIDO, 2011)

Tabela 4: Matriz de Correlação de Pearson entre as variáveis latentes ou constructos

\begin{tabular}{|c|c|c|c|c|c|c|c|}
\hline Constructos & EsF. P\&D & ESTRATÉGIA & InOv. Proc. & Inov. Prod. & ORG. IND. & TAMANHO & Uso EMA \\
\hline Esf. P\&D & 1,0000 & & & & & & \\
\hline Estratégia & 0,2721 & 0,7944 & & & & & \\
\hline Inov. Proc. & 0,3646 & 0,6361 & 0,7388 & & & & \\
\hline Inov. Prod. & 0,6657 & 0,7380 & 0,7290 & 0,8024 & & & \\
\hline Org. Ind. & 0,3589 & 0,4982 & 0,5655 & 0,5781 & 1,0000 & & \\
\hline Tamanho & 0,1336 & 0,6002 & 0,3153 & 0,3976 & 0,2412 & 0,8646 & \\
\hline Uso EMA & 0,1931 & 0,7124 & 0,3773 & 0,6313 & 0,5749 & 0,5786 & 0,7241 \\
\hline
\end{tabular}

Obs.: Raízes quadradas da Variância Média Extraída (VME) evidenciadas na diagonal destacadas em negrito.

Fonte: Dados da pesquisa

Os resultados encontrados mostram ainda que a correlação existente entre as variáveis latentes "inovação de processos" e "inovação de produtos" (Tabela 4) está muito próxima dos valores encontrados na análise da validade discriminante do constructo teórico de inovação de processos. Isso pode indicar que quando os índices de inovação de produtos são altos nas empresas, os índices de inovação de processos também serão.

Em relação à validação do modelo estrutural proposto por Ferreira, Moulang e Hendro (2010), nota-se nos resultados obtidos para os índices de R2 (Tabela 3), que o menor $\mathrm{R} 2$ entre os constructos analisados foi de 0,0740, para a variável latente esforço em P\&D.
O uso do sistema EMA obteve um coeficiente de explicação de 0,6162 , e os constructos de inovação de produtos e inovação de processos, um R2 de 0,8075 e 0,4583 , respectivamente. Os achados da pesquisa apontam para um coeficiente de explicação superior ao encontrado no estudo de Ferreira, Moulang e Hendro (2010), o que confirma a validade do modelo teórico proposto pelos autores.

A Figura 2 apresenta os relacionamentos estruturais das variáveis e os resultados dos coeficientes beta da análise de caminhos e os respectivos coeficientes de explicação dos constructos teóricos investigados, adequados nos aspectos de validade convergente, validade discriminante e confiabilidade.

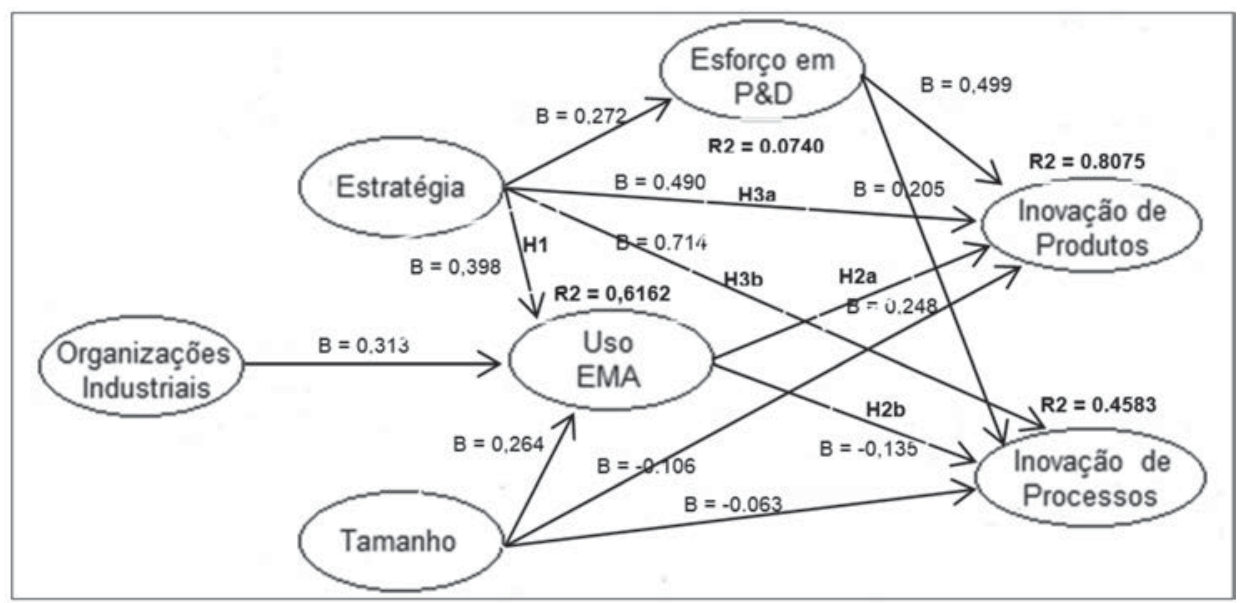

Figura 2: Análise de caminhos do modelo estrutural PLS

Fonte: Dados da pesquisa 
Convergente com a metodologia desenvolvida por Ferreira, Moulang e Hendro (2010), utilizou-se um código de identificação das empresas, para diferir as indústrias com maior risco ambiental, das demais empresas. Tal diferenciação é recomendada pelos autores para distinguir as empresas com alto índice de risco ambiental das demais. Em tais empresas a utilização de sistemas de contabilidade de gestão ambiental não deve ser considerada uma inovação. Dessa forma, as indústrias metalúrgicas foram codificadas com o número 1 e as demais empresas, com o número 0 . Os resultados dos testes de hipóteses realizados na pesquisa são evidenciados na Tabela 5 .

Tabela 5: Resultados dos testes de hipóteses realizados

\begin{tabular}{|c|c|c|c|c|c|}
\hline HiPÓTESES & $\begin{array}{c}\text { Coefic. } \\
\text { Beta }\end{array}$ & $\begin{array}{c}\text { ERro } \\
\text { PADRÃo }\end{array}$ & $\begin{array}{l}\text { VALOR } \\
\text { DE T }\end{array}$ & PVALUE & $\begin{array}{c}\text { RELAÇão } \\
\text { DiRETA }\end{array}$ \\
\hline $\begin{array}{l}\text { H1: Há uma associação positiva entre a es- } \\
\text { tratégia prospectora e uso EMA. }\end{array}$ & 0,398 & 0,1075 & 3,7006 & 0,00023 & Aceita \\
\hline $\begin{array}{l}\text { H2a: Há uma associação positiva entre o } \\
\text { uso EMA e inovação de produtos. }\end{array}$ & 0,248 & 0,0634 & 3,9095 & 0,00010 & Aceita \\
\hline $\begin{array}{l}\text { H2b: Há uma associação positiva entre o } \\
\text { uso EMA e inovação de processos. }\end{array}$ & $-0,135$ & 0,1250 & 1,0760 & 0,28219 & $\begin{array}{l}\text { Não su- } \\
\text { portada }\end{array}$ \\
\hline $\begin{array}{l}\text { H3a: Há uma associação positiva entre a estraté- } \\
\text { gia prospectora e inovação dos produtos. }\end{array}$ & 0,490 & 0,0645 & 11,2228 & 0,00000 & Aceita \\
\hline $\begin{array}{l}\text { H3b: Há uma associação positiva entre a estraté- } \\
\text { gia prospectora e inovação dos processos. }\end{array}$ & 0,714 & 0,0552 & 12,9625 & 0,00000 & Aceita \\
\hline
\end{tabular}

Fonte: Dados da pesquisa

A hipótese $\mathrm{H} 1$ testada na pesquisa estabelece uma associação positiva entre a estratégia prospectora e o uso do sistema EMA. A utilização de sistemas de contabilidade de gestão ambiental fornece informações que podem contribuir positivamente para a inovação de produtos nas empresas. Logo, em empresas que almejam a liderança de mercado espera-se que elas sejam mais propensas à adoção do sistema EMA. Os resultados encontrados na Tabela 5 mostram que tal relacionamento foi suportado nesta pesquisa. A análise da correlação realizada para estss constructos (Tabela 4) e os resultados do teste de hipóteses (Tabela 5) indicam que a relação entre as duas variáveis é estatisticamente significativa.

A hipótese $\mathrm{H} 2 \mathrm{a}$ da pesquisa também pode ser aceita neste estudo, pois há uma associação positiva entre o uso do sistema EMA e a inovação de produtos. Hansen e Mowen (2005) já destacavam que a inovação de produtos é um benefício obtido pelas indústrias em decorrência da utilização do sistema EMA. Apesar da confirmação dessa hipótese (H2a), o mesmo relacionamento não pode ser confirmado em relação à inovação de processos, hipótese $\mathrm{H} 2 \mathrm{~b}$ testada no estudo. $\mathrm{O}$ coeficiente beta encontrado para esse relacionamento foi negativo (Tabela 5). No entanto, nota-se na análise das correlações dos constructos de "inovação de processos" e "inovação de produtos" (Tabela 4), que há uma tendência das empresas pesquisadas com elevados índices de inovação de produtos, também, apresentar altos índices de inovação de processos.

Em relação às demais hipóteses testadas na pesquisa, verifica-se, pelos resultados encontrados na Tabela 5, que há associação positiva entre a estratégia prospectora e a inovação dos produtos $(\mathrm{H} 3 \mathrm{a})$ e a estratégia prospectora $e$ a inovação de processos (H3b). Empresas com estratégia prospectora enfatizam a importância do desenvolvimento de produtos e processos inovadores (MILES; SNOW, 1978; GIMENEZ et al., 1999) e a adoção de práticas de gerenciamento por atividades. (GOSSELIN, 1997)

É possivel observar ainda, nos resultados da pesquisa (Figura 2), que a variável contingencial tamanho possui associação positiva com o uso do sistema EMA nas empresas pesquisadas. Entretanto, tal associação não foi observada na relação dos constructos de tamanho e inovação de produtos, bem como de tamanho e inovação de processos, o que também pode estar relacionado à forte correlação existente entre os 
constructos de inovação de processos e inovação de produtos (Tabela 4), os quais sugerem a tendência desta relação apenas em empresas com maiores níveis de inovação de produtos.

Em relação aos constructos teóricos investigados na pesquisa (Figura 1), verificou-se a validade do modelo teórico descrito por Ferreira, Moulang e Hendro (2010). As evidências encontradas neste estudo, convergentes com os achados de Hansen e Mowen (2005), sugerem a existência de associação positiva entre o uso do sistema de contabilidade de gestão ambiental e inovação em empresas industriais de grande porte. De acordo com Ferreira, Moulang e Hendro (2010), o uso do sistema EMA ajuda as empresas a reconhecer os efeitos ambientais de suas atividades operacionais. Em virtude de seus potenciais benefícios, o uso desse sistema contribui para a melhoria do processo decisório das empresas, razão pela qual sua utilização vem se expandindo. (SCHALTEGGER; BURRITT, 2000; BURRITT, 2004; DE BEER; FRIEND, 2006; FERREIRA; MOULANG; HENDRO, 2010; CHRIST; BURRITT, 2013)

\section{Considerações Finais}

Este estudo objetivou identificar a relação entre práticas de contabilidade de gestão ambiental e inovação, fundamentado no modelo teórico de Ferreira, Moulang e Hendro (2010), em empresas industriais de grande porte estabelecidas no Rio Grande do Sul, Brasil. Para tanto, realizou-se uma pesquisa descritiva, por meio de levantamento e abordagem quantitativa. A amostra da pesquisa compreendeu 21 empresas industriais de grande porte, localizadas no Estado do Rio Grande do Sul, que atuam em diferentes segmentos econômicos.

Sobre as práticas de EMA adotadas nas empresas investigadas, os resultados da pesquisa mostram que elas se utilizam de sistemas de contabilidade de gestão ambiental, principalmente com práticas de: introdução ou melhoria de gerenciamento de custos relacionados ao meio ambiente, desenvolvimento e uso de indicadores de desempenho relacionados ao meio ambiente, identificação de custos relacionados ao meio ambiente e sua respectiva alocação para processos de produção e produtos, análise de melhorias de produtos e análise de estoques de produtos.
Convergente com os achados de estudos anteriores, os resultados da pesquisa indicam que os benefícios obtidos pelas empresas com a utilização do sistema EMA não se restringem aos elementos econômico-financeiros. Nas empresas pesquisadas, percebeu-se, entre os principais benefícios, melhoria na reputação da empresa, melhoria na tomada de decisões, identificação de novas oportunidades de negócio, geração de inovação de processos e melhoria dos processos de produção. A adoção desses sistemas auxilia também os gestores na melhoria do custeio de produtos e, por consequência, melhoria na decisão de preços.

Em relação aos constructos teóricos investigados na pesquisa, verificou-se a validade do modelo. Os resultados do estudo indicam um alto poder de explicação do modelo teórico de análise do uso do sistema EMA pelas empresas pesquisadas, e suas relações com as variáveis de inovação de produtos e inovação de processos. Evidenciam associação positiva entre a estratégia prospectora e o tamanho da empresa, em relação ao uso de sistemas de contabilidade de gestão ambiental. A estratégia prospectora utilizada nas empresas pesquisadas influencia ainda o esforço em pesquisa e desenvolvimento, inovação de produtos e inovação de processos. As variáveis inovação de processos e inovação de produtos também apresentaram forte correlação.

Assim, conclui-se que há associação positiva entre o uso de sistemas de contabilidade de gestão ambiental e inovação nas empresas pesquisadas. No entanto, apesar dos potenciais benefícios, que podem ser obtidos com a utilização de sistemas de contabilidade de gestão ambiental, verificou-se que nem todas as empresas pesquisadas os utilizam. Os resultados do estudo evidenciam que entre as empresas pesquisadas, as maiores, ambientalmente sensíveis e com estratégia ambiental definida são mais propensas à implementação de sistemas de contabilidade de gestão ambiental.

Possivelmente, a obtenção de um maior número de informações, de natureza física e monetária, qualificaria o processo decisório dos gestores, e contribuiria para uma melhor avaliação dos processos de gestão da empresa como um todo. A escassez ou falta de informações pode induzir os gestores a decisões equivocadas, comprometendo assim a competitividade, além de acarretar eventuais perdas para as empresas. Especula-se que a qualidade do processo decisório 
dos gestores seja prejudicada sem a presença desses sistemas, o que implica novas pesquisas.

Os resultados encontrados fornecem evidências que estimulam a realização de estudos adicionais. Apesar dos potenciais benefícios previstos na literatura com a adoção desses sistemas, instiga o fato de nem todas as empresas pesquisadas utilizá-los. Os baixos indicadores observados nos benefícios atribuídos para a utilização do sistema EMA, advindos da atração de equipe de trabalho de melhor qualidade, redução de custos de seguros e redução do custo de capital, estimulam a realização de novas pesquisas. As evidências encontradas sugerem que tais benefícios não seriam determinantes para a implementação de sistemas de contabilidade de gestão ambiental. É oportuna ainda a replicação deste estudo em uma amostra maior, com o intuito de abranger empresas de médio e grande porte localizadas em outras regiões do país. A investigação empírica desses constructos em outro ambiente pode contribuir para a confirmação ou refutação dos achados desta pesquisa.

\section{REFERÊNCIAS}

ADAMS, C.; ZUTSHI, A. Corporate social responsibility: why business should actress ponsiblyandbe accountable. Australian Accounting Review, Australia, v. 14, n. 3, p. 31-40, 2004.

BARBIERI, J. C. Gestão ambiental empresarial: conceitos, modelos e instrumentos. São Paulo: Saraiva 2006.

BENNETT, M.; BOUMA, J.J.; WOLTERS, T. Environmental Management Accounting: informational and institutional developments. Eco-Efficiency in Industryand Science, USA, v. 9, p. 1-319, Springer, 2002.

BENNETT, M.; RIKHARDSSON, P.; SCHALTEGGER, S. Adopting environmental management accounting: EMA as a value-adding activity. Eco-Efficiency in Industryand Science, USA, v. 12, p. 1-14, 2003.

BIDO, D. S. et al. Indicadores formativos na modelagem em equações estruturais com estimação via PLS-PM: Como lidar com a multicolinearidade entre eles? In: EnEPQ, 2., 2009, Curitiba. Anais... Rio de Janeiro: ANPAD, 2009. CD-ROM.
BIDO, D. S. et al. Articulação entre as aprendizagens individual, grupal e organizacional: um estudo no ambiente industrial. Revista de Administração Mackenzie, São Paulo, v. 11, n. 2, p. 68-95, mar.-abr., 2010.

BRAMER, S.; BROOKS, C.; PAVELIN, S. Corporate social performance and stocks returns: UK evidence from disaggregates measures. Financial Management, USA, v. 35, n. 3, p. 97-116, 2006.

BRAZ, J. L. P. et al. Contabilidade ambiental: proposta para a evidenciação do resultado do desempenho social e ambiental de uma autarquia municipal do interior do Estado de São Paulo no ano de 2007. Revista de Administração e Inovação, São Paulo, v. 6, n. 3, p. 7993, 2009.

BURRITT, R. L. Environmental management accounting: roadblocks on the way to the green anpleasant land.

Business Strategy and the Environmental, USA, v. 13, n. 1, p. 13-32, Jan.-Feb. 2004.

BURRITT, R.; HAHN, T.; SCHALTEGGER, S. Towards a comprehensive framework for environmental management accounting-links between business actors and environmental management accounting tools.

Australian Accounting Review, Australia, v. 12, n. 2, p. 39-50, 2002.

BURRITT, R. L.; SAKA, B. C. Environmental management accounting applications and eco-efficiency: case studies from Japan. Journal of Cleaner Production, USA, v. 14, n. 14, p. 1.262-1.275, 2006.

CHRIST, K. L.; BURRITT, R. L. Environmental management accounting: the significance of contingent variables for adoption. Journal of Cleaner Production, USA, v. 41, p. 163-173, 2013.

DAVILA, T. An empirical study on the drivers of management control systems design in new product development. Accounting, Organizations and Society, USA, v. 25, n. 4/5, p. 383-409, 2000.

DAVILA, A.; FOSTER, G.; LI, M. Reasons for management control systems adoption: Insights from product development choice by early-stage entrepreneurial companies. Accounting, Organizations and Society, USA, v. 34, n. 3/4, p. 322-347, 2009. 
DE BEER, P.; FRIEND, F. Environmental accounting: a management tool for enhancing corporate environmental and economic performance. Ecological Economics, USA, v. 58, n. 3, p. 548-60, 2006.

\section{DEEGAN, C. M. Environment Management}

Accounting: an introduction and case studies for Australia. Sydney: Institute of Chartered Accountants in Australia, 2003.

DRAKE, A. R.; HAKA, S. F.; RAVENSCROFT, S. P. Cost system and incentive structure effects on innovation, efficiency and profitability in teams. The Accounting Review, USA, v. 74, n. 3, p. 323-345, 1999.

FERREIRA, A.; MOULANG, C.; HENDRO, B.

Environmental management accounting and innovation: an exploratory analysis. Accounting, Auditing \&

Accountability Journal, USA, v. 23, n. 7, p. 920-948, 2010.

FROST, G. R.; WILMSHURST, T. D. The adoption of environment-related management accounting: ananalysis of corporate environment sensitivity. Accounting Forum, USA, n. 24, p. 345-365, 2000.

GALE R. Environmental management accounting as a reflexive modernization strategy in cleaner production. Journal of Cleaner Production, USA, v. 14, n. 14, p. 1.228-1.236, 2006.

GIBSON, K. C.; MARTIN, B. A. Demonstrating value through the use of environmental management accounting. Environmental Quality Management, USA, v. 13, n. 3, p. 45, Spring, 2004.

GIL, A. C. Como elaborar projetos de pesquisa. 4. ed. São Paulo. Atlas, 2002.

GIMENEZ, F. A. P. et al. Estratégia em pequenas empresas: uma aplicação do modelo de Miles e Snow.

Revista de Administração Contemporânea, [on-line], v. 3, n. 2, p. 53-74, 1999.

GOSSELIN, M. The effect of strategy and organizational structure on the adoption and implementation of activitybased costing. Accounting, Organizations and Society, USA, v. 22, n. 2, p. 105-122, 1997.

HAIR, J. F. et al. Multivariate data analysis with readings. 5. ed. New Jersey: Prentice Hall, 1998.
HAMBRICK, D.C. Some tests of the effectiveness and functional attributes of Miles and Snow's strategic types. Academyof Management Journal, USA, v. 26, n. 1, p. 5-26, 1983.

HANSEN, D. R.; MOWEN, M. M. Environmental cost management, management accounting. Mason, $\mathrm{OH}$ : Thomson-South-Western, 2005. p. 490-526.

MARSHALL, R. S.; BROWN, D. Corporate environmental reporting: what's in a metric? Business Strategy and the Environment, USA, v. 12, n. 2, Mar.-Apr., 2003.

MARTENDAL, A. et al. Contabilidade ambiental: nível de conhecimento dos profissionais contábeis da Grande Florianópolis. Enfoque: Reflexão Contábil, Maringá, PR, v. 32, n. 1, p. 29-47, jan.-abr., 2013.

MARTIN, B. Environmental accounting. Financial Management, USA, p. 36-37, Apr. 2007.

MASANET-LLODRA, M. Environmental management accounting: a case study research on innovative strategy. Journal of Business Ethics, USA, v. 68, n. 4, p. 393408, 2006.

MENEZES, E. A. C.; GUIMARÃES, T. A.; BIDO, D. S. Dimensões da aprendizagem em organizações: validação do Dimensions of the Learning Organization Questionnaire (DLOQ) no contexto brasileiro. Revista de Administração Mackenzie, São Paulo, v. 12, n. 2, p. 4-29, mar.-abr., 2011.

\section{MILES, R. E.; SNOW, C. C. Organizational strategy,} structure and process. New York: McGraw-Hill, 1978.

NISIYAMA, E. K.; OYADOMARI, J. C. T. Sistemas de controle gerencial e o processo de inovação. Revista de Administração e Inovação, São Paulo, v. 9, n. 1, p.106125, jan.-mar., 2012.

NUNES, J .P. O.; PFITSCHER, E. D.; UHLMANN, V. O. Um aporte ao sistema contábil gerencial ambiental: segunda geração de indicadores. Revista de Gestão Social e Ambiental, São Paulo, v. 5, n. 2, p. 154-171, 2011.

OYADOMARI, J. C. T. et al. Sistemas de controle gerencial: estudo de caso comparativo em empresas inovadoras no Brasil. Revista Universo Contábil, Blumenau, SC, v. 6, n. 4, p. 21-34, out.-dez., 2010. 
PAMPLONA, V. et al. Gestão e contabilidade ambiental: estudo de caso em instituição hospitalar. Contabilidade, Gestão e Governança, Brasília, DF, v. 14, n. 2, p. 3-17, 2011.

REVELLINO, S.; MOURITSEN, J. The multiplicity of controls and the making of innovation. European Accounting Review, USA, v. 18, n. 2, p. 341-369, 2009.

ROGERS, G.; KRISTOF, J. Reducing operation and product costs through environmental accounting.

Environmental Quality Management, USA, v. 12, n. 3, Spring, 2003.

SCAVONE, G. M. Challenges in internal environmental management reporting in Argentina. Journal of Cleaner Production, USA, v. 14, n. 14, p. 1.276-1.285, 2006.

SCHALTEGGER, S. et al. Environmental Management accounting for cleaner production. Eco-Efficiency in Industry and Science, USA, v. 24, p. 3-28, Springer, 2009.

SEIFFERT, M. E. B.; LOCH, C. Systemic thinking in environmental management: support for sustainable development. Journal of Cleaner Production, USA, v. 13, n. 12, p. 1.197-1.202, 2005.

STANISKIS, J. K.; STASISKIENE, Z. Environmental management accounting in Lithuania: exploratory study of current practices, opportunities and strategic intents. Journal of Cleaner Production, USA, v. 14, p. 1.2521.261, 2006.

VASILE, E.; MAN, M. Current dimension of environmental management accounting. Procedia - Social and

Behavioral Sciences, USA, v. 62, p. 566-570, 2012.

VELLANI, C. L.; RIBEIRO, M. S. Sistema contábil para gestão da ecoeficiência empresarial. Revista Contabilidade \& Finanças, São Paulo, v. 20, n. 49, p. 25-43, 2009. 